\title{
Schematherapie bei Essstörungen - Ein integrativer Ansatz zur Verbesserung des Therapieoutcomes
}

\section{Schematherapy in Eating Disorders - An Integrative Approach to Improve the Outcome}

\author{
Autoren
}

Christina Archonti ${ }^{1}$, Martina de Zwaan

Institute

${ }^{1}$ Psychotherapeutische Praxis, Frankfurt am Main

${ }^{2}$ Klinik für Psychosomatik und Psychotherapie, Medizinische Hochschule Hannover

\section{Schlüsselwörter \\ - Anorexia nervosa \\ - Bulimia nervosa \\ - Schematherapie \\ - Erlebnisaktivierende Methoden}

Keywords

- anorexia nervosa

- bulimia nervosa

- schema therapy

- experiential techniques eingereicht 17. April 2016 akzeptiert 6. Mai 2016

\section{Bibliografie}

DOI http://dx.doi.org/ 10.1055/s-0042-108721

Psychother Psych Med 2016; 66: 275-279

(c) Georg Thieme Verlag KG Stuttgart · New York ISSN 0937-2032

Korrespondenzadresse

Dr. Christina Archonti, Dipl.-Psych. Dr. rer.med. Psychotherapeutische Praxis Kettenhofweg 94 60325 Frankfurt am Main kontakt@praxis-archonti.de

\section{Zusammenfassung}

Die Behandlung von Essstörungen stellt trotz der vorhandenen verhaltenstherapeutischen und psychodynamischen Therapiekonzepte weiterhin eine therapeutische Herausforderung dar. Essstörungen gehen mit einer hohen komorbiden Prävalenz von Persönlichkeitsstörungen und weiterer komorbider Achse-I-Störungen sowie einer hohen Abbruch- und Rückfallrate einher und weisen bei Anorexia nervosa die höchste Mortalitätsrate unter den psychiatrischen Störungen auf. Häufig gelingt es trotz der selbstinitiierten Motivation der Patientin nicht, ein normales Essverhalten zu entwickeln, das Gewicht $\mathrm{zu}$ regulieren und die zugrunde liegenden dysfunktionalen Verhaltensmuster und Kognitionen zu verändern. Vorgestellt wird eine Adaptation der Schematherapie nach Young, integriert in eine evidenzbasierte kognitive Verhaltenstherapie, mit dem Ziel die Therapiemotivation und das -outcome mittels erlebnisorientierter Methoden zu unterstützen.

\section{Essstörungen}

$\nabla$

Essstörungen stellen trotz der vorhandenen verhaltenstherapeutischen und psychodynamischen Therapiekonzepte und partieller Erfolge der entsprechenden Therapieformen [1,2] weiterhin eine therapeutische Herausforderung dar. Hierzu gehören eine teils stark ich-syntone und damit sehr veränderungsresistente Symptomatik, störungsspezifische Folgen der Erkrankung, die höchste Mortalität unter allen psychischen Erkrankungen (bezogen auf die Anorexia nervosa), hohe Raten an psychiatrischer Komorbidität sowie eine hohe Chronifizierungs- und Rückfallrate [3].

*Die wesentliche Form wird aufgrund des Geschlechterverhältnisses der Störung gewählt

\section{Abstract \\ $\nabla$}

Despite evidence-based psychotherapeutic treatment approaches such as cognitive behavioral therapy and psychodynamic therapy eating disorders still pose a challenge to therapists and patients alike. Eating disorders are associated with a high comorbid prevalence of personality disorders and other psychological axis-I-disorders, show highdrop-out rates and relapse rates and anorexia nervosa has the highest mortality rate compared to all psychiatric disorders. Even self-motivated patients frequently fail to achieve the treatment goals like developing a normal eating behavior, gaining weight, and changing the underlying dysfunctional behavioral patterns and cognitions. We will present a schematherapeutic approach with experiential methods, integrated in evidence-based CBT, with the intention to improve motivation and therapeutic outcome. 
chische Störungen sich therapieresistent zeigten [4]. Die fehlende Ansprechbarkeit auf therapeutische Verfahren wurde als Ausdruck maladaptiver Schemata, die die Patienten in ihren früheren Beziehungserfahrungen erworben haben, verstanden. Die daraus resultierenden maladaptiven Schema-Bewältigungsreaktionen führen zu einem Verhalten, das regelmäßig den Kriterien einer Persönlichkeitsstörung entspricht und sich als besonders ausgeprägt, unflexibel oder wenig angepasst bis rigide zeigt.

Das therapeutische Modell wurde um ein Repertoire an Interventionen erweitert, um diese zugrunde liegenden den Therapieprozess blockierenden strukturellen Störungen effektiver behandeln zu können.

Die Schematherapie wurde für Patienten mit therapieresistenten psychischen Störungen entwickelt. Eine Grundannahme ist, dass maladaptive Schemata den Therapieprozess blockieren. Durch Interventionen sollen diese Störungen behandelt werden.

\section{Das schematherapeutische Konzept}

Das schematherapeutische Modell und das daraus abgeleitete Vorgehen beinhalten als Kernkomponenten die Art der Befriedigung der zentralen psychischen Grundbedürfnisse [5], daraus folgenden Emotionen und der im Erleben eingeprägten Folgen frustrierter (kindlicher) Grundbedürfnisse. Diese negativen emotionalen Schemata bestimmen fortan im Sinne einer erworbenen Reaktionsbereitschaft (trait) die Wahrnehmung, Bewertung und Handlungsimpulse in allen weiteren sozialen Interaktionen [4].

Den Schemaaktivierungen folgen wiederum 3 mögliche Bewältigungsmuster, die sich zeigen in der Erduldung eines scheinbar gegebenen und nicht zu ändernden Schicksals, der Vermeidung der (schemaaktivierenden) Situationen oder deren Kompensation, als eine Art aktives und überzogenes Gegenhandeln - ohne das Schema in seiner Wirksamkeit zu ändern [6].

Wahrnehmung, Bewertung und Handlungsimpulse der Patienten werden durch negative emotionale Schemata bestimmt.

\section{Das schematherapeutische Modusmodell \\ $\nabla$}

Die Kombination von Schemata und daraus abgeleitete Bewältigungsstrategien führt in der Gegenwart zu beschreibbaren Erlebniszuständen (Modi), die sich in folgende psychische Konstrukte unterteilen lassen:

Kindmodi: Basale Emotionen als Ausdruck erlebter unbefriedigter Grundbedürfnisse sowie des unmittelbaren Erlebens eigener Möglichkeiten und Lösungen. Das Erleben in diesem Modus ist sehr verzerrt und geht mit einer Reihe von Über- und Unterschätzungen eigener Möglichkeiten oder der Bedeutung der Situation und des Gegenübers einher.

Dysfunktionale „innere Elternmodi“: bewertende, entwertendstrafende, überkritische Selbstinstruktionen als Internalisierungen der (impliziten und expliziten) Anforderungen und Bewertungen signifikanter Bezugspersonen, gesellschaftlicher oder kultureller Gegebenheiten und Normen.

Maladaptive Bewältigungsmodi: positiv oder negativ verstärkte Lösungsversuche aus der Biografie zur Reduktion mögli- cher Inkonsistenzen zwischen Bedürfnissen (Kind) und Anforderungen (Innere Elternmodi) in sozialen Interaktionen. Es kann zwischen den 3 grundlegenden Möglichkeiten der bindungsorientierten Unterwerfung, des vermeidenden Rückzugs oder des selbstbehauptungsorientierten »Kämpfens» (Überkompensation) unterschieden werden.

Der gesunde Erwachsenenmodus: wird als selbstfürsorgliche, regulierende und vermittelnde Instanz verstanden. Der Modus umfasst eine Reihe von Kompetenzen, die zu einem lösungsorientierten, realitätsbezogenen und ausgeglichenen Umgang des Individuums mit seinen Bedürfnissen und den Anforderungen aus der Umwelt führen. Zentral ist die Fertigkeit, widersprüchliche Bedürfnisse wie Bindung vs. Autonomie mittels guter Kompromisse zu integrieren und die Bedürfnisbilanz im Mittel ausgeglichen zu halten.

Die angenommene Dynamik zwischen diesen Modi wird im folgenden Modell dargestellt ( $\bullet$ Abb. 1).

Das Erarbeiten dieses Modells zählt zu den kognitiven Elementen der schematherapeutischen Arbeit. Mittels der dann folgenden emotionsgeleiteten Methoden, der Imagination und der Stühlearbeit (Dialog auf mehreren Stühlen), werden diese Einheiten erlebnisorientiert exploriert und mit Symptomen in Beziehung gesetzt. Im letzten Schritt wird, noch während der emotionalen Exposition, neues und lösungsorientiertes Verhalten verankert [6].

Ein weiteres und sehr bedeutsames Element stellt die therapeutische „Nachbeelterung“ (Reparenting) dar, das kognitive, aber noch mehr am Erleben orientierte Validieren und Vermitteln selbstfürsorglicher, die Grundbedürfnisse und die Person des Patienten achtender, wertschätzender, gesunder „selbstbezogener“ Verhaltensweisen, zu denen oftmals keinerlei Bezug und Zugang mehr besteht.

Die Kombination von Schemata und daraus abgeleitete Bewältigungsstrategien führt zu beschreibbaren Erlebniszuständen (Modi). Diese stehen in einer dynamischen Verbindung zueinander. Anhand emotionsgeleiteter Methoden können die Modi exploriert und mit Symptomen in Verbindung gesetzt werden.

\section{Schematherapie bei Essstörungen}

Die Schematherapie bei Essstörungen stellt kein neues störungsspezifisches Verfahren dar, sondern die Implementierung eines am Erleben orientierten Vorgehens in die bisherige Therapie. Es kann in seiner dargelegten Form bei allen Formen von Essstörungen angewendet werden (bei Patientinnen mit Anorexie und Bulimie ebenso wie bei Patienten mit kompensatorischem Essverhalten und Essanfällen mit Übergewicht) und bietet damit ein störungs- oder vielmehr von der aktuellen Ausprägung der Essstörung (vgl. [7]) unabhängiges therapeutisches Vorgehen. Jegliche maladaptive Ausprägung des Essverhaltens kann in der schemabasierten Fallkonzeption als Teil eines Bewältigungsmodus integriert werden.

Das erlebnisorientierte Vorgehen dient nicht dem direkten Verändern der zugrunde liegenden Symptomatik, sondern orientiert sich an den Entstehungs- und aufrechterhaltenden Bedingungen und der Behandlung der Hintergrundproblematik.

Dabei werden die psychopathologischen Ursachen wie geringes Selbstwertgefühl und negatives Selbstkonzept sowie die Folgen, die sich aus der Kompensation ergeben (wie Überwertigkeit von Figur und Gewicht, Hungern oder auch Essen) in die Therapie integriert. 


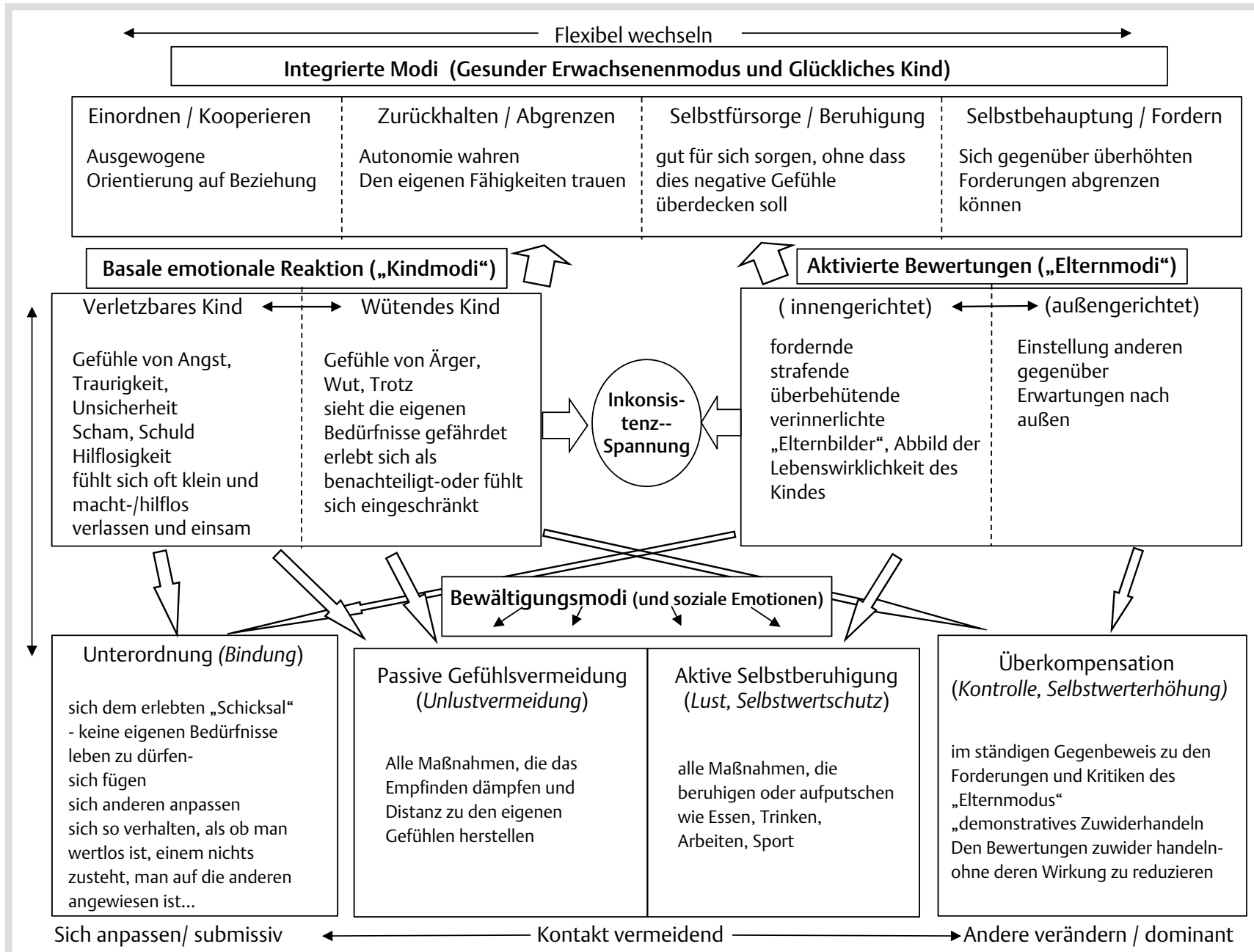

Abb. 1 Modusmodell.

Es kann eine sinnvolle und weiterführende Ergänzung der KVT $[3,8]$ und der erweiterten KVT für Essstörungen (CBT-E) [7] für Patientinnen darstellen, welche von dem vorgestellten Therapiemanagement nicht oder nicht ausreichend profitieren. Es stellt auch eine hilfreiche Ergänzung im Therapieplan einer Erstbehandlung dar.

Die Schematherapie bei Essstörungen kann eine sinnvolle Ergänzung der KVT und CBT-E darstellen. Sie dient nicht dem direkten Verändern der Symptomatik, sondern orientiert sich an den Entstehungs- und aufrechterhaltenden Bedingungen und der Behandlung der Hintergrundproblematik.

Mit diesem in Bezug auf die KVT der Essstörungen erweiterten Vorgehen soll das Folgende erreicht werden:

- Das frühe Erarbeiten eines Störungsmodells unter Einbeziehung der der Essstörung zugrunde liegenden Hintergrundproblematik, negativer Selbst- und Beziehungsschemata und daraus ableitbarer Modi, die das Erreichen der zu Beginn bestimmten Therapieziele der KVT behindern und damit therapieschädigend wirken.

- Verbesserung der Motivation für die Therapie, speziell wenn die ersten Schwierigkeiten auftreten, sowie ein konstruktiver Umgang mit Motivationsproblemen, um Therapieabbrüchen vorzubeugen.
- Ein Verständnis für die Funktionalität einzelner, die Essstörung begründender, Verhaltensweisen.

Im Klärungsprozess können sowohl die Patientin als auch ihre Psychotherapeutin besser verstehen, dass das gestörte Essverhalten, in welcher Form es auch immer gerade auftritt, stets Folge und nicht Ursache der Probleme der Patientin ist, wenngleich es im Verlauf eine eigene Störungsdynamik mit aufrechterhaltenden Bedienungen entwickelt und entsprechend den Richtlinien [3] auch parallel behandelt werden muss.

Das schematherapeutische Vorgehen hat zum Ziel, die handlungsleitenden kognitiv-emotionalen Prozesse in der Sitzung emotional zu aktualisieren und die Patienten dabei zu unterstützen, sich von diesen zu distanzieren und alternative Verhaltensweisen zu entwickeln. Im weiteren Verlauf soll die Anwendung der Verhaltensalternativen gefördert und eingeübt werden.

\section{Integration der Schematherapie in den Behandlungsplan}

Das Behandlungsmanagement orientiert sich an dem einer ambulanten Langzeittherapie, wobei die schematherapeutischen Elemente auch im Rahmen einer stationären Therapie von Beginn einbezogen werden können, um die erforderlichen Therapiemodule der KVT zu unterstützen und die schematherapeuti- 
Tab. 1 Behandlungsplan nach Archonti, Roediger, de Zwaan [10].

\author{
Therapieplan \\ Erstgespräch, Anamnese, Diagnostik, Klärung der Motivation \\ Klärung und Verbesserung der Therapiemotivation- Erstellen eines Störungs- \\ modells, Psychoedukation zu den körperlichen und psychischen Folgen der \\ Essstörung \\ Klärung und Aufbau der Motivation \\ Normalisierung des Körpergewichts und der Ernährung -Therapievertrag mit \\ Festlegen des Zielgewichts, Interventionen zur Verhinderung von Essattacken, \\ Einführung von Ess- und Gedankenprotokolle \\ Fertigkeiten der Gefühlsregulation (Skills I) \\ Fertigkeiten zum Aufbau und Führen zwischenmenschlicher Beziehungen (Skills II)
}

\author{
Erweiterung \\ Erhebung der Schemata nach Young, Erklärung zu negativen Sche- \\ mata, Informationen zur Schematherapie und der Arbeit mit den Modi \\ Information über das schematherapeutische Bedingungsmodell, \\ Erstellen einer schematherapeutischen Verhaltensanalyse (Modus- \\ modell) \\ Einbezug des Modusmodells, Dialog auf 2 Stühlen \\ Imagination, Teilearbeit („Stühlearbeit“), Innerer Dialog, Tagebuch- \\ karte
}

sche Arbeit einzuleiten. Der Behandlungsplan kann $\bullet$ Tab. 1 entnommen werden.

\section{Schematherapeutische Interventionen}

Grundsätzlich lassen sich die Interventionen in der Schematherapie in kognitive und emotionsorientierte, erlebnisaktivierende Methoden unterteilen [9].

\section{Fallkonzeption}

$\nabla$

Die Fallkonzeption wird wie eine klassische Verhaltensanalyse der KVT zusammen mit der Patientin auf einem Flipchart oder auf einem Vordruck gemeinsam erstellt. Sie stellt auf eine validierende Art die Reaktion der Patientin auf verschiedene, meist sehr alltägliche Lebensereignisse dar und macht die Funktion der verschiedenen Elemente der Essstörung (Gewicht und Figur, Nahrungsaufnahme, verschiedene gegenregulatorische Maßnahmen) vor der Hintergrundproblematik der negativen Schemata deutlich.

Das Ziel ist, zunächst ein kognitives Verständnis der Modi sowie damit assoziierter Gefühle und ein erstes Modell zu erarbeiten, auf das sich die weitere schematherapeutisch gestützte Arbeit beziehen kann. Die meisten Patientinnen erleben das auf den ersten Blick recht komplex wirkende Modell als nachvollziehbar, validierend und sehr entlastend. Dieser Effekt ist umso stärker, je komplexer die Beeinträchtigung durch zugrunde liegende strukturelle Anteile ist bzw. je ausgeprägter eine zugrunde liegende Persönlichkeitsstörung ist, da es die Zerrissenheit der Patienten sehr anschaulich abbildet.

Die Visualisierung der Modi sowie der damit assoziierten Emotionen wirkt auf die meisten Patienten entlastend.

\section{Schemamemo}

Das schematherapeutisch geleitete Gefühlsprotokoll [10] stellt eine modifizierte, um die Elemente der Schematherapie und speziell der erlebnisaktivierenden Arbeit ergänzte Form der Gefühls- und Gedankenprotokolle der KVT dar. Es eignet sich v.a. zur Verbesserung der Selbststeuerung, nachdem erste erlebnisaktivierende Methoden und speziell eine Imagination durchgeführt wurden und die Patientin ihre Modi verstanden hat.

Ziel des schematherapeutisch geleiteten Gefühlsprotokolls ist die Verbesserung der emotionalen Wahrnehmung und des Aus- drucks, der Differenzierung zwischen Auslösern und eigenem Verhalten, eine Selbstvalidierung sowie das Benennen und Einüben von Verhaltensalternativen. Zur Verbesserung der emotionalen Wahrnehmung und speziell der entsprechenden Modi werden körperliche Anteile der Emotionen einbezogen.

\section{Imagination}

Die Imagination stellt zusammen mit den Dialogen auf Stühlen das Herzstück der schematherapeutischen Arbeit dar. Es gehört zu den grundlegenden Techniken in den Einzeltherapien, die einen Zugang zu den Bedürfnissen und Emotionen der Patientin ermöglichen.

Der zentrale Schritt bei den Imaginationsübungen besteht darin, aus den semantisch-verbalen Informationsverarbeitungs- und Gedächtnissystemen auf eine körpernah-emotional-episodische Ebene zu wechseln, was einen direkteren Zugang zu Assoziationen und Bildern aus dem biografischen Gedächtnis und dem sog. „float back“ ermöglicht [6]. Die assoziierte Kindheitsszene steht für das Lebensgefühl des Kindes (als Beispiel für das frustrierte oder verletzte Grundbedürfnis) und bewirkt ein am Erleben orientiertes Verständnis für die erworbenen - heute hinderlichen oder problematischen - Bewältigungsstrategien, zu denen auch jede Art gestörten Essverhaltens gehört.

Im Idealfall erleben die Patientinnen, dass ihre heutigen Gefühle denen in der bildlich-emotional-assoziativ gefundenen biografischen Szene ähnlich sind, auch wenn die Szene kognitiv keinerlei Bezug zur heutigen Lebenssituation aufweist. Dies macht erleb- und fassbar, dass die heutigen Gefühle stärker durch alte Schemata bewirkt werden als durch die aktuelle Auslösesituation. Aspekte des gestörten Essverhaltens werden, ohne dass ihre Notwendigkeit bzw. Schädlichkeit diskutiert werden muss, erlebnisorientiert mit den frustrierten Grundbedürfnissen in Beziehung gesetzt. Die Bedeutung von Gewicht und Figur für die Regulation der eigenen Gefühle wird unmittelbar erfahren und so der Weg zur Veränderung geebnet [10].

In der sog. Überschreibung (Rescripting) werden auf das Selbst bezogene und bei Bedarf auch nahrungsbezogene dysfunktionale Glaubenssätze erlebnisnah korrigiert. Die Wirkung der Entmachtung und Versorgung (Reparenting) wird unmittelbar körperlich von den Patienten erfahren und bewirkt als zentrale Elemente eine Veränderung des Erlebens der heutigen Szene und eine deutliche Erweiterung der Handlungskompetenzen der Betreffenden. Die Intervention erfordert therapeutische Erfahrung, Sicherheit im Umgang mit der Intervention und im 
Umgang mit Gefühlen und nach Möglichkeit auch das sichere Anwenden verschiedener Validierungsstufen [11].

Die Imagination ist ein zentraler Teil der schematherapeutischen Arbeit. Sie gehört zu den grundlegenden Techniken in den Einzeltherapien, die einen Zugang zu den Bedürfnissen und Emotionen der Patientin ermöglichen.

\section{Stuhldialoge \\ $\nabla$}

Die Modusdialoge auf mehreren Stühlen ermöglichen, in der Gegenwart verschiedene innere Anteile (Modi) der Patienten „auseinanderzusetzen“ und getrennt und spezifisch zu bearbeiten. Die anfangs im Vordergrund stehenden Bewältigungsmodi werden von den Patientinnen i.d. R. als ich-synton erlebt - sei es die Überzeugung, zu dick und unansehnlich zu sein, oder jene, nur als besonders schlanke Frau eine Chance auf Zugehörigkeit und Anerkennung zu haben oder sich das Essen verdienen zu müssen, gepaart mit dem Gedanken, sowieso „nie genug“ dafür getan zu haben. Störungsübergreifend persistiert häufig der „innere Kritiker" mit sehr vernichtenden, selbstabwertenden Kognitionen. Auch hier besteht die zentrale Intervention in einer emotionalen, mit den jeweiligen Anteilen in Verbindung stehenden Aktivierung, einer Distanzierung, Heraussetzen und Entmachten der toxisch wirkenden verinnerlichten „Kritiker“ und ihrer Glaubenssätze im Moment ihrer Aktivierung. Sehr einprägsam gestaltet sich für Patienten, wenn sie anhand eines leeren Stuhles, der als Platzhalter für z.B. einen entwertenden und strafenden Anteil steht und symbolisch vor die Tür gestellt werden soll, an ihrer Angst spüren, wie sie von ihren eigenen Überzeugungen im Griff gehalten werden - und zu erleben, wie sie ihre Gefühle anders als durch Vermeiden oder Selbstberuhigen (bzw. Hungern, unkontrolliertes Essen oder Sichübergeben u.a.) regulieren können (ähnlich wie in anderen Expositionsverfahren).

Mithilfe von Stuhldialogen lassen sich die Modi der Patienten voneinander getrennt bearbeiten und für die Patienten visualisieren.

\section{Fazit für die Praxis}

Vorgestellt wurde eine kurze Übersicht der schematherapeutischen Arbeit und deren Integration in eine störungsspezifische KVT-Essstörungstherapie. Bei der Integration der schematherapeutischen Arbeit wird ein therapeutisches Vorgehen empfohlen, in dem der Einsatz der Schematherapie mit aufeinander bezogenen Schritten geplant wird. In der klinischen Arbeit zeigt sich eine hohe Akzeptanz der Patientinnen für das Vorgehen, die häufig berichten, über die Arbeit ein besseres Verständnis ihrer Essstörung bekommen, sich effektiver von deren Grundsätzen distanzieren und Wege zur Veränderung wirkungsvoller umsetzen zu können. Der schematherapeutische Zugang zeigt sich als sehr hilfreich für den Wirkfaktor der therapeutischen Beziehung sowie in der Veränderung negativer Schemata und der sie begleitenden emotionalen Störungen.
Interessenkonflikt: Die Autoren geben an, dass kein Interessenkonflikt besteht.

\section{Literatur}

1 Herpertz-Dahlmann B, Schwarte R, Krei M et al. Day-patient treatment after short inpatient care versus continued inpatient treatment in adolescents with anorexia nervosa (ANDI): a multicentre, randomized, open-label, non-inferiority trial. Lancet 2014; 383: 1222-1229

2 Zipfel S, Wild B, Friedrich HC et al. Focal psychodynamic therapy, cognitive behaviour therapy, and optimised treatment as usual in outpatients with anorexia nervosa (ANTOP study): randomized controlled trial. Lancet 2013; 383: 127-137

3 Herpertz S, de Zwaan M, Zipfel S. Handbuch der Essstörungen und Adipositas. Heidelberg: Springer; 2015

4 Young JE, Klosko JS, Weishaar ME. Schematherapie - ein praxisorientiertes Handbuch. Paderborn: Junfermann; 2005

5 Grawe K, Donati R, Bernauer F. Psychotherapie im Wandel. Von der Konfession zur Profession. Göttingen: Hogrefe; 1994

6 Roediger E. Schematherapie. Grundlagen - Anwendungen - Perspektiven. Stuttgart: Schattauer; 2016

7 Fairburn CG. Kognitive Verhaltenstherapie und Essstörungen. Stuttgart: Schattauer; 2012

8 Legenbauer T, Vocks S. Manual der Kognitiven Verhaltenstherapie bei Anorexie und Bulimie. Heidelberg: Springer; 2014

9 Jacob G, Arntz A. Schematherapie in der Praxis. Weinheim: Beltz; 2015

10 Archonti C, Roediger E, de Zwaan M. Schematherapie bei Essstörungen. Weinheim: Beltz; 2016

11 Linehan MM. DBT Skills Training Manual. New York: Guilford Publications; 2014 